\title{
Mutations in Main Protease of SARS CoV-2 Decreased Boceprevir Affinity
}

\author{
Ekrem Akbulut ${ }^{*}$ \\ https://orcid.org/ 0000-0002-7526-9835 \\ ${ }^{1}$ Malatya Turgut Ozal University, Department of Bioengineering, Malatya, Turkey. \\ Editor-in-Chief: Alexandre Rasi Aoki \\ Associate Editor: Daniel Fernandes
}

Received: 2020.12.23; Accepted: 2021.05.25.

*Correspondence: ekremakbulut@gmail.com; Tel.: +905333312316 (E.A.).

\section{HIGHLIGHTS}

- Boceprevir is a potential therapeutic that targets the main protease of SARS-CoV-2.

- Mutations change the inhibitory binding pattern in the catalytic domain of the main protease.

- Mutations of main protease of SARS-CoV-2 cause a decrease in boceprevir affinity.

\begin{abstract}
The coronavirus disease 2019 (COVID-19) caused by severe acute respiratory syndrome coronavirus 2 (SARS-CoV-2) is a global health emergency. The main protease ( $\mathrm{M}^{\text {pro }}$ ) is crucial for the life cycle of coronaviruses. Boceprevir is a potential inhibitor and drug candidate for the $\mathrm{M}^{\text {pro }}$ of SARS-CoV-2. In this study, changes in the protein structure of the $\mathrm{M}^{\text {pro }}$ due to mutations in SARS-CoV-2 and the effects of these changes on boceprevir affinity, an important potential therapeutic agent, were investigated. The mutations were analyzed with RDP4 and MegaX. A three-dimensional model of mutant $\mathrm{M}^{\text {pro }}$ was generated by ProMod3. Qualitative Model Energy Analysis, ProSA, and MolProbity tools were used for structural validation and modeling of the wild-type and mutant $\mathrm{M}^{\text {pro }}$ proteins. Topological differences of the wild-type and mutant $\mathrm{M}^{\text {pro }}$ were calculated with the $\mathrm{i}$-Tasser TM-Score. Molecular docking was performed using AutoDock 4.2. Functional dynamic structure models were created with DynOmics. Seven mutations (L89F, K90R, P108S, A191V, T224A, A234V and S254F) were detected in the Mpro of SARS-CoV-2. The mutations caused a decrease in the affinity of boceprevir, a potential protease inhibitor. The boceprevir was docked to the active site of $\mathrm{M}^{\text {pro }}$, and the binding energies were -10.34 and $-9.41 \mathrm{kcal}^{\mathrm{mol}}{ }^{-1}$ for the wild-type and the mutant, respectively. The Debye-Waller factors calculated by elastic network model analysis were 0.58 and $0.64 \AA^{2}$ for the wild-type $M^{\text {pro }}$ and mutant $M^{\text {pro }}$, respectively. Mutations in structures that are important drug targets for SARS-CoV-2 may render existing therapeutics ineffective in its treatment.
\end{abstract}

Keywords: boceprevir affinity; drug validity; main protease; mutation; SARS-CoV-2.

\section{INTRODUCTION}

Severe acute respiratory syndrome coronavirus-2 (SARS-CoV-2) is the etiological agent responsible for the global coronavirus disease 2019 (COVID-19) outbreak [1]. SARS-CoV-2 emerged in China at the end of 2019 and spread all over the world in a short time [2]. SARS-CoV-2 killed 3.1 million people in the past sixteen months [3]. Apart from SARS-CoV-2, it is known that there are six types of coronaviruses that cause diseases in humans. Four of these (229E, OC43, NL63, and HKU1) are common and typically cause flu symptoms [4]. 
The other two strains-severe acute respiratory syndrome coronavirus (SARS-CoV) and Middle East respiratory syndrome coronavirus (MERS-CoV)-are of zoonotic origin and deadly pathogens [5]. The high prevalence, wide distribution, genomic diversity, and high recombination potential of coronaviruses reveal the fact that the new members of this virus family, which appear from time to time, may infect humans in the future $[5,6]$.

Studies on vaccines for prophylaxis of COVID-19 have recently been successful, but a specific therapeutic drug molecule is still being developed. Generally, there are studies to reposition the drugs used for treatment of different diseases for the treatment of COVID-19. Although favipiravir, ribavirin, chloroquine, merimepodib, and remdesivir, which are used in the treatment of different diseases, are not specific therapeutic drugs for COVID-19, they are used despite their limited contribution to the healing process of the disease [7-9]. Therefore, the development of specific drugs is urgent.

SARS-CoV-2 is a positive-polarity RNA virus. The genomes of RNA viruses show a high risk of mutation. One of the important reasons affecting the spread rate and mortality rate of the disease is mutations in SARSCoV-2 strains [10]. More than 12,000 mutations have been identified in the SARS-CoV-2 genome to date. While some of these mutations do not cause any changes in the virulence properties of the virus, some of them may have negative results in many factors ranging from virulence features to treatment sensitivity [1113].

The SARS-CoV-2 genome has a single-chain, 29.9 kilobase size, organization of 12 structural proteins $[14,15]$. The major open reading frame (ORF) 1ab encodes for two overlapping polyproteins (pp1a and pp1ab), which are cleaved into 16 nonstructural proteins by the main protease ( $\mathrm{M}^{\text {pro }}$ or known as $3 \mathrm{CL}^{\text {pro }}$ ) and the papain-like protease $\left(\mathrm{PL}^{\mathrm{pro}}\right)$ [16-19]. These nonstructural proteins regulate the production of SARS-CoV2 basic structural (spike, envelope, membrane and nucleocapsid) and accessory proteins. Therefore, $M^{\text {pro }}$ is crucial for the life cycle of coronaviruses [20]. Mpro cleaves pp1ab at 11 distinct sites. It is considered an important antiviral drug target because it is structurally different from human proteases [9,21]. On the other hand, $\mathrm{PL}$ pro recognizes the $\mathrm{C}$-terminal sequence of ubiquitin. For this reason, $\mathrm{PL}$ pro inhibitors derived from the substrate have the disadvantage of also inhibiting host-cell deubiquitinases [20]. SARS-CoV-2 Mro is known to be similar to other coronaviral main proteases. In particular, the high structural similarity in the active site enables the use of previous gains for treatment in SARS-CoV-2 $\mathrm{M}^{\text {pro }}$ [22-24]. Viral proteases are promising targets for many different viruses, including hepatitis $\mathrm{C}$ virus (HCV) and human immunodeficiency virus. Since there are no specific antiviral drugs clinically approved to combat SARS-CoV-2, the use of clinically approved drugs for different diseases is urgently required to treat COVID-19 [25]. Boceprevir is a peptidomimetic ketoamide serine protease tripeptide inhibitor used in the treatment of HCV genotype 1 [26,27]. Although boceprevir is not a specific drug for SARS-CoV-2, it is thought to be a potential drug candidate for SARSCoV-2 due to its inhibition success on the protease of the HCV $[25,28-30]$. Many studies have shown that boceprevir also effectively inhibits the SARS-CoV-2 $\mathrm{M}^{\text {pro }}[25,31]$.

The urgent demand for the development of drugs effective against SARS-CoV-2 requires the use of in silico methods for rapid screening of potential therapeutic molecules. Many in silico studies have suggested potential inhibitors against proteins of SARS-CoV-2 [32-34]. In this study, changes in the protein structure of the main protease due to mutations in SARS-CoV-2 North American isolates and the effects of these changes on boceprevir affinity, an important potential therapeutic agent for SARS-CoV-2, were investigated by in silico techniques. This study aims to provide critical information on the optimization and design of stronger inhibitors against SARS-CoV-2.

\section{MATERIALS AND METHODS}

\section{Sequence and Mutation Data}

In this study, information on the nucleotide and protein sequence of 319 isolates from the North American continent belonging to the SARS-CoV-2 Mpro was obtained from the NCBI Virus database (NCBI 2020). The reference $M^{\text {pro }}$ accession code is YP_009725301.1. The protein sequence information of 319 isolates was aligned with the MAFFT (v7.471) multiple sequence alignment program FFT-NS-i algorithm [35-37]. The scoring matrix BLOSUM 80 was chosen for the amino acid sequences [38]. A value of 2.0 was used for gap opening penalty. The mutated residues were analyzed with the RDP4 and MegaX $[39,40]$. 


\section{Homology Model of Mutant Protein}

A three-dimensional model of mutant-type SARS-CoV-2 $\mathrm{M}^{\text {pro }}$ was generated by the method of homology modeling using Swiss-Model [41]. 6zru (RCSB protein data bank code) was selected as the template. Boceprevir, dimethyl sulfoxide, and water molecules were removed from the template structure. Models were built based on the target-template alignment using ProMod3 (ver3.1.1) [42]. Qualitative Model Energy Analysis (QMEAN), ProSA, and MolProbity tools were used for structural validation and modeling of the wildtype and mutant $\mathrm{M}^{\text {pro }}$ proteins [43-45]. Secondary structure components (random coils, beta strands, and alpha helices) of the $\mathrm{M}^{\text {pro }}$ protein were defined with PSIPRED [46]. Superimpose and conformational analyses of the wild-type and mutant proteins were performed with UCSF Chimera (ver1.14) and PyMOL (ver2.4.1) [47]. Topological differences of the wild-type and mutant $\mathrm{M}^{\text {pro }}$ were calculated with the $\mathrm{i}$-Tasser TM-Score and root mean square deviation (RMSD) algorithm $[48,49]$.

\section{Docking}

The modeled structures of both wild-type and mutant were used as targets, and boceprevir (CID 10324367) was used as the ligand for molecular docking using AutoDock 4.2 [50]. Kollman charges were added to the $\mathrm{M}^{\text {pro }}$ wild-type and mutant models. Gasteiger partial charges were applied to the ligands. Docking was performed with a grid dimension of $68 \times 100 \times 98(-15.989,-30.532,8.404)$ with a grid spacing of $0.375 \AA$ around the binding pocket. Docking simulations were performed with the Lamarckian genetic algorithm (LGA) [51]. The main selected LGA parameters were 100 runs, $2.7 \times 10^{4}$ generations, and 300 population size. A maximum of $2.5 \times 10^{7}$ energy evaluations was applied for each experiment. The results were clustered according to binding energy scores using a tolerance of $2.0 \AA$ RMSD. Docking results were visualized with Discovery SV (ver20.1, DDS Biovia).

\section{Dynamic Network Models}

Functional dynamic structure models in the protein structure before and after mutations were created with the DynOmics portal. Coarse-grained models for conformational dynamic analysis for the wild-type and mutant proteins were created with the Anisotropic Network Model and the Gaussian Network Model (GNM) $[52,53]$.

\section{Protein Stability and Ligand Affinity Analysis}

Analysis of changes in protein stability was performed using DynaMut2 [54], SDM [55], mCSM stability [56] and DUET [57]. The change in boceprevir affinity before and after the mutation was evaluated using the CSM-lig program [58].

\section{RESULTS}

Mutations of the SARS-CoV-2 $\mathrm{M}^{\text {pro }}$ caused a decrease in the affinity of boceprevir, a potential protease inhibitor for SARS-CoV-2. Seven mutations (L89F, K90R, P108S, A191V, T224A, A234V, and S254F) in the $\mathrm{M}^{\text {pro }}$ of SARS-CoV-2 were detected in the North American isolates (Table 1).

The mutation data were processed on the $\mathrm{M}^{\text {pro }}$ sequence information of SARS-CoV-2. The mutant $\mathrm{M}^{\text {pro }}$ was modeled. For the mutant $\mathrm{M}^{\text {pro }}$ model, the QMEAN score was -0.03 , the Ramachandran favored score was $98.3 \%$, the Ramachandran outlier was $0.33 \%$, and the MolProbity score was 0.9 . The Z-score of the mutant model was -7.06 , and the model was found in the native protein range that was identified by $\mathrm{X}$-ray crystallography (Figure 1).

Protein stability analyses data showed that the mean distance change was $3.81 \AA$, the total energy change was $-4.22 \mathrm{kcal} / \mathrm{mol}$, and the change in protein stability was $-1.06 \mathrm{kcal} / \mathrm{mol}$. While the residues $89,108,191,234$ and 254 decreased the molecular flexibility, the residues number 90 and 224 increased the molecular flexibility. While mutations at residues $89,90,108,191,224$ and 254 caused destabilization of the protein, the mutation at residue 234 contributed to protein stabilization (Table 2). Boceprevir affinities were 10.0 and $9.4\left(-\log _{10}(\mathrm{KD} / \mathrm{Ki})\right)$ in the wild-type and mutant proteins, respectively. 
Table 1. Mutations of SARS-CoV-2 Mpro.

Mutant site Mutant sequence ID

\begin{tabular}{ll}
\hline & QNR99561.1, QNN95411.1, QNN95747.1, QNN96047.1, QNL12792.1, QNL12876.1, \\
& QNN95351.1, QNL36356.1, QNL36368.1, QNK39067.1, QNK39031.1, QNK39043.1, \\
& QNK39055.1, QNR99357.1, QNR99393.1, QNR99429.1, QNN95807.1, QNN95483.1, \\
& QN L>F \\
& QNN96071.1, QNN96119.1, QNS00107.1, QNL12684.1, QNL13128.1, QNR54217.1 \\
& QNR99501.1, QNR99549.1, QNS00095.1, QNS00119.1, QNL12504.1, QNL12516.1, \\
& QNL10944.1, QNL12648.1, QNL12540.1, QNN95423.1, QNL12576.1, QNL12588.1, \\
& QNL12528.1 \\
\hline 90 K>R & QNL12744.1, QNL11508.1, QNL11280.1 \\
\hline 108 P>S & QNR99345.1, QNS00143.1, QNS00155.1, QNN95711.1, QNN95891.1 \\
\hline 191 A>V & QNR99357.1, QNR99393.1, QNR99441.1, QNR99477.1 \\
\hline 224 T>A & QNL36356.1, QNL36368.1, QNK39067.1, QNK39031.1, QNK39043.1, QNK39055.1 \\
\hline 234 A>V & QNN95351.1, QNR99357.1, QNR99393.1, QNR99441.1, QNR99477.1 \\
\hline 254 S>F & QNL10956.1, QNL11028.1, QNL11112.1
\end{tabular}

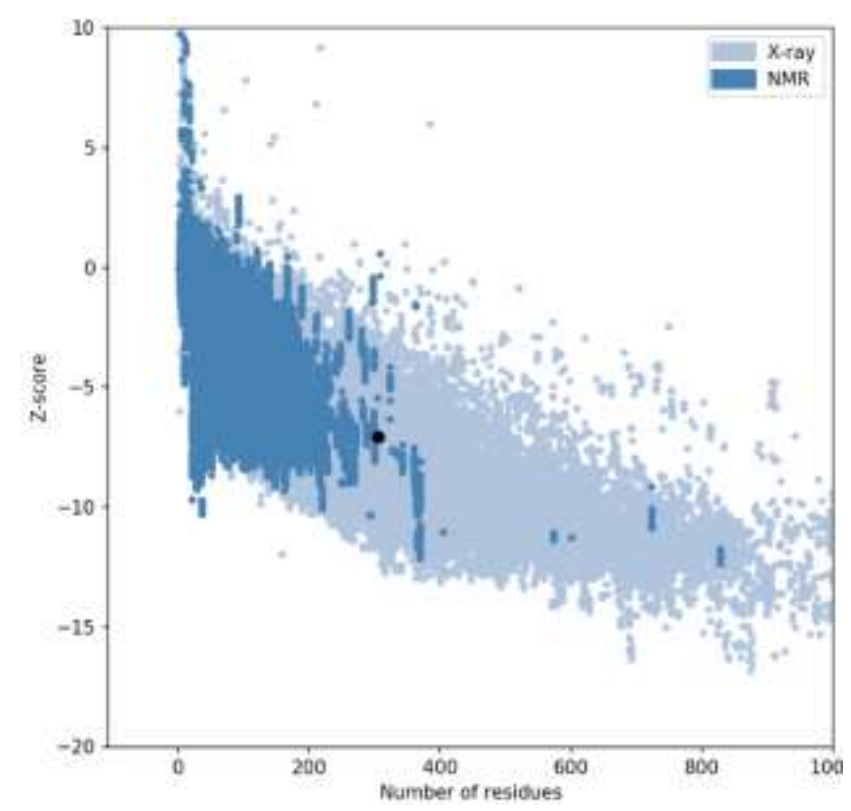

Figure 1. Z score - Model quality of the mutant SARS-CoV-2 Mpro.

Superimposition of the mutant and the wild-type structures showed that the RMSD value was $0.072 \AA$ and the TM-Score was 0.9 . Boceprevir was docked to the active site of the $\mathrm{M}^{\text {pro: }}$ : the binding energies were between -10.34 and $-6.14 \mathrm{kcal}^{\mathrm{mol}}{ }^{-1}$ and between -9.41 and $-4.98 \mathrm{kcal}^{\mathrm{mol}}{ }^{-1}$ for the wild type and mutant type, respectively. While the wild-type $\mathrm{M}^{\text {pro }}$ interacted with boceprevir in 32 different conformations, the mutant $\mathrm{M}^{\text {pro }}$ interacted in 35 different conformations (Table 3).

The lowest binding energy and inhibitor concentration for boceprevir were $-10.34 \mathrm{kcal}^{\mathrm{mol}} \mathrm{m}^{-1}$ and 0.03 $\mu \mathrm{M}$ for the wild-type $\mathrm{M}^{\text {pro }}$, respectively. For the conformation at this binding energy, boceprevir formed a hydrogen bond with the wild-type $\mathrm{M}^{\text {pro }}$ between the Gly143:N and Boc:O36 residues, between the His41:NE2 and Boc:O4 residues, between the Cys134:SG and Boc:N11 residues, and between Thr26:O and Boc:N37 residues. The boceprevir formed a $\mathrm{C}-\mathrm{H}$ bond with the wild-type $\mathrm{M}^{\text {pro }}$ between the His 164:O and Boc:C19 residues. Boceprevir formed alkyl interactions between the Met49, Met165, and Pro168 residues and pi-alkyl interactions between residues His41 and His163 (Figure 2). 
Table 2. Change in SARS-CoV-2 Mpro stability.

$\Delta \Delta \mathbf{G}\left(\mathrm{kcal} . \mathrm{mol}^{-1}\right)$

$\Delta \Delta S_{\text {Vib }}\left(\mathrm{kcal} \mathrm{mol}^{-1} . \mathrm{K}^{-1}\right)$

\begin{tabular}{cccccccccccc} 
wild & position & mutant & $\begin{array}{c}\text { RSA } \\
(\%)\end{array}$ & mCSM & SDM & DUET & ENCoM & DynaMut & ENCoM & Flex. & Stability \\
\hline L & 89 & $\mathbf{F}$ & 0.0 & -1.851 & -0.66 & -1.803 & 0.182 & -0.108 & -0.228 & Dec. & Destabilizing \\
\hline K & 90 & $\mathbf{R}$ & 47.6 & -0.887 & -0.21 & -0.554 & -0.040 & 0.272 & 0.049 & Inc. & Destabilizing \\
\hline $\mathbf{P}$ & 108 & $\mathbf{S}$ & 32.4 & -1.48 & -0.55 & -1.428 & 0.142 & -0.585 & -0.177 & Dec. & Destabilizing \\
\hline A & 191 & $\mathbf{V}$ & 98.4 & -0.327 & -0.04 & -0.014 & 0.272 & 0.440 & -0.340 & Dec. & Destabilizing \\
\hline T & 224 & $\mathbf{A}$ & 67.0 & -0.675 & 0.15 & -0.434 & -0.646 & -0.240 & 0.807 & Inc. & Destabilizing \\
\hline A & 234 & $\mathbf{V}$ & 0.8 & 0.204 & -1.03 & 0.384 & 0.421 & -0.242 & -0.527 & Dec. & Stabilizing \\
\hline S & 254 & $\mathbf{F}$ & 20.8 & -1.032 & 1.02 & -0.568 & 0.185 & 0.315 & -0.231 & Dec. & Destabilizing \\
\hline
\end{tabular}

Dec.: Decrease, Inc.: Increase, Flex: Flexibility, RSA: Residue relative solvent accessibility, SDM: Site direct mutator, $\Delta \Delta \mathrm{S}_{\mathrm{Vib}}: \Delta$ Vibrational entropy energy between wild-type and mutant.

Table 3. Docking results ranked by energy.

\begin{tabular}{|c|c|c|c|c|c|c|c|c|c|}
\hline \multirow[b]{2}{*}{ Row } & \multicolumn{2}{|c|}{ wild } & \multicolumn{2}{|c|}{ mutant } & \multirow[b]{2}{*}{ Row } & \multicolumn{2}{|c|}{ wild } & \multicolumn{2}{|c|}{ mutant } \\
\hline & $\begin{array}{l}\text { Binding } \\
\text { energy } \\
\text { kcal } / \mathrm{mol}\end{array}$ & $\begin{array}{l}\mathrm{Ki}^{\mathrm{e}} \\
\mu \mathrm{M}\end{array}$ & $\begin{array}{l}\text { Binding } \\
\text { energy } \\
\text { kcal } / \mathrm{mol}\end{array}$ & $\begin{array}{l}\mathbf{K i}^{\mathrm{e}} \\
\mu \mathrm{M}\end{array}$ & & $\begin{array}{c}\text { Binding } \\
\text { energy } \\
\text { kcal } / \mathrm{mol}\end{array}$ & $\begin{array}{l}\mathrm{Ki}^{\mathrm{e}} \\
\mu \mathrm{M}\end{array}$ & $\begin{array}{l}\text { Binding } \\
\text { energy } \\
\text { kcal } / \mathrm{mol}\end{array}$ & $\begin{array}{l}\mathrm{Ki}^{\mathrm{e}} \\
\mu \mathrm{M}\end{array}$ \\
\hline 1 & -10.34 & 0.03 & -9.41 & 0.13 & 19 & -7.24 & 4.97 & -7.10 & 6.23 \\
\hline 2 & -9.65 & 0.09 & -8.63 & 0.47 & 20 & \begin{tabular}{|l|}
-7.21 \\
\end{tabular} & 5.21 & $\begin{array}{l}-7.04 \\
\end{array}$ & 6.89 \\
\hline 3 & -9.31 & 0.15 & -8.61 & 0.49 & 21 & -7.14 & 5.84 & -6.99 & 7.54 \\
\hline 4 & $\begin{array}{l}-9.03 \\
\end{array}$ & 0.24 & -8.57 & 0.52 & 22 & -7.12 & 6.03 & -6.89 & 8.86 \\
\hline 5 & -8.45 & 0.64 & -8.39 & 0.71 & 23 & -7.04 & 6.86 & -6.85 & 9.50 \\
\hline 6 & \begin{tabular}{|l|}
-8.36 \\
\end{tabular} & 0.75 & -8.33 & 0.78 & 24 & -6.97 & 7.80 & -6.82 & 10.02 \\
\hline 7 & $\begin{array}{l}-8.24 \\
\end{array}$ & 0.92 & -8.04 & 1.29 & 25 & -6.87 & 9.18 & -6.70 & 12.25 \\
\hline 8 & -8.17 & 1.03 & $\begin{array}{l}-7.98 \\
\end{array}$ & 1.42 & 26 & -6.83 & 9.88 & $\begin{array}{l}-6.68 \\
\end{array}$ & $\begin{array}{l}12.63 \\
\end{array}$ \\
\hline 9 & -8.08 & 1.19 & -7.97 & 1.43 & 27 & -6.67 & 12.85 & -6.55 & 15.93 \\
\hline 10 & -7.97 & 1.43 & -7.85 & 1.77 & 28 & -6.58 & 15.09 & -6.51 & 16.89 \\
\hline 11 & $\begin{array}{l}-7.82 \\
\end{array}$ & 1.85 & -7.46 & 3.42 & 29 & -6.56 & 15.61 & -6.43 & 19.51 \\
\hline 12 & -7.75 & 2.08 & -7.45 & 3.43 & 30 & -6.51 & 17.05 & -6.35 & 22.31 \\
\hline 13 & -7.62 & 2.58 & -7.35 & 4.08 & 31 & -6.37 & 21.47 & -6.16 & 30.70 \\
\hline 14 & -7.55 & 2.91 & -7.34 & 4.19 & 32 & -6.14 & 31.71 & -6.10 & 33.87 \\
\hline 15 & $\begin{array}{l}-7.49 \\
\end{array}$ & 3.22 & \begin{tabular}{|l|}
-7.31 \\
\end{tabular} & 4.41 & 33 & & & -5.81 & 54.83 \\
\hline 16 & -7.43 & 3.56 & -7.30 & 4.47 & 34 & & & -5.72 & 63.39 \\
\hline 17 & -7.33 & 4.22 & -7.20 & 5.26 & 35 & & & -4.98 & 225.43 \\
\hline 18 & -7.28 & 4.64 & -7.10 & 6.22 & & & & & \\
\hline
\end{tabular}

Kie- estimated minimum inhibitor concentration

On the other hand, the lowest binding energy and inhibitor concentration for boceprevir were -9.41 kcal.mol ${ }^{-1}$ and $0.13 \mu \mathrm{M}$ for the mutant $\mathrm{M}^{\text {pro }}$, respectively. For the conformation at this binding energy, boceprevir formed a hydrogen bond with the mutant $\mathrm{M}^{\text {pro }}$ between the CYS145:N and Boc:O1 residues, between the Cys145:SG and Boc:N11 residues, and between the Thr26:O and Boc:N37 residues. Boceprevir formed a $\mathrm{C}-\mathrm{H}$ bond with the mutant $\mathrm{M}^{\text {pro }}$ between the His164:O and Boc:C19 residues. Boceprevir formed alkyl interactions between the Met49 and Met165 residues and pi-alkyl interactions between the His41 and His 163 residues (Figure 3).

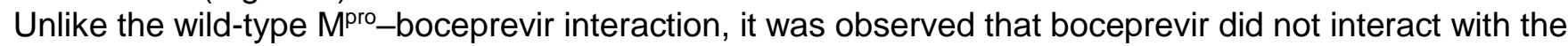
mutant type, as shown by the absence of hydrogen bonding with His41 and alkyl interaction with Pro168. A well-regulated water molecule appears to stabilize the existing covalent conformation in the binding pocket 
in both wild and mutant protein-boceprevir interaction by coordinating hydrogen bonds between the amide group of $\alpha$-ketoamide and the backbone oxygen of Thr26.

The Debye-Waller factors calculated by elastic network model analysis were 0.58 and $0.64 \AA^{2}$ for the wild-type $\mathrm{M}^{\text {pro }}$ and mutant $\mathrm{M}^{\text {pro }}$, respectively. Although the collective motion (Figure 4) of the protein did not change, the fluctuation distances decreased. The root mean square fluctuation (RMSF) (Figure 5) and flexibility values (Table 2) of residues showed that the protein transformed into a rigid structure after the mutations.
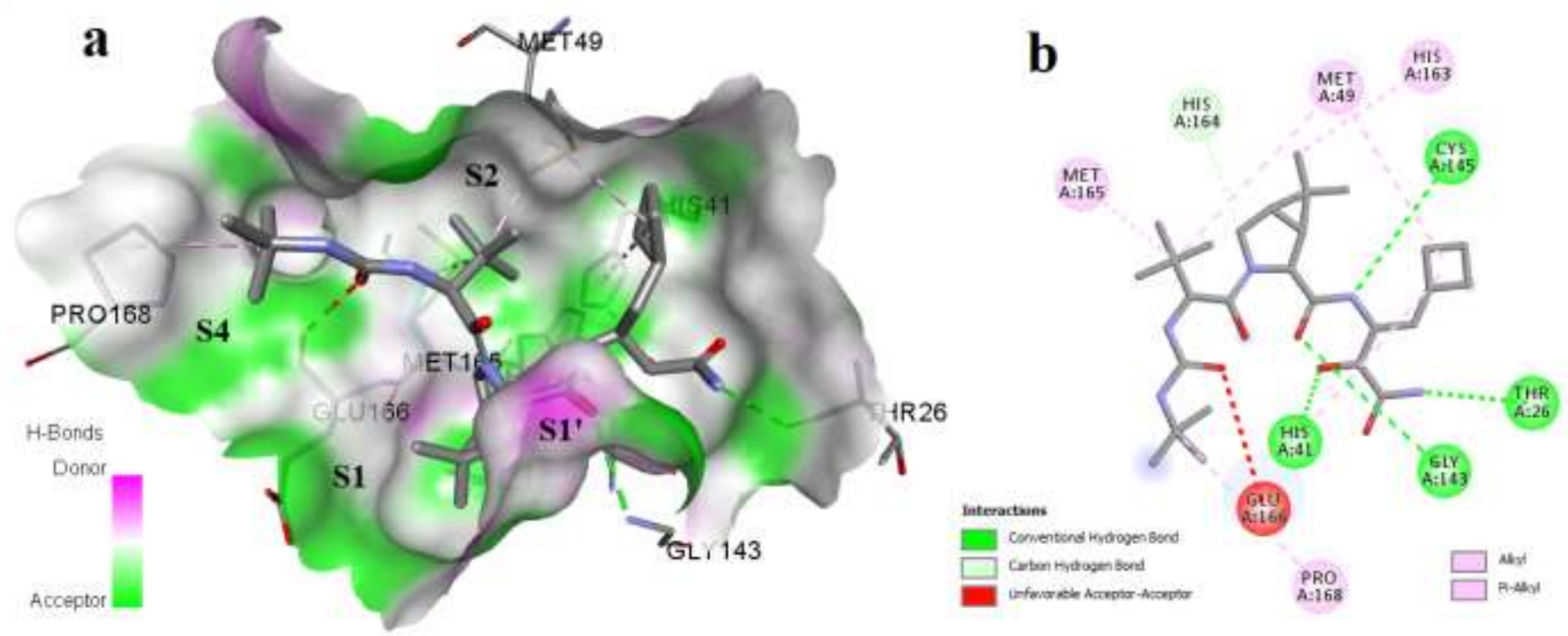

Figure 2. Bond interaction of boceprevir with wild-type SARS-CoV-2 Mpro.

a) Surface representation, b) Diagram representation

b)

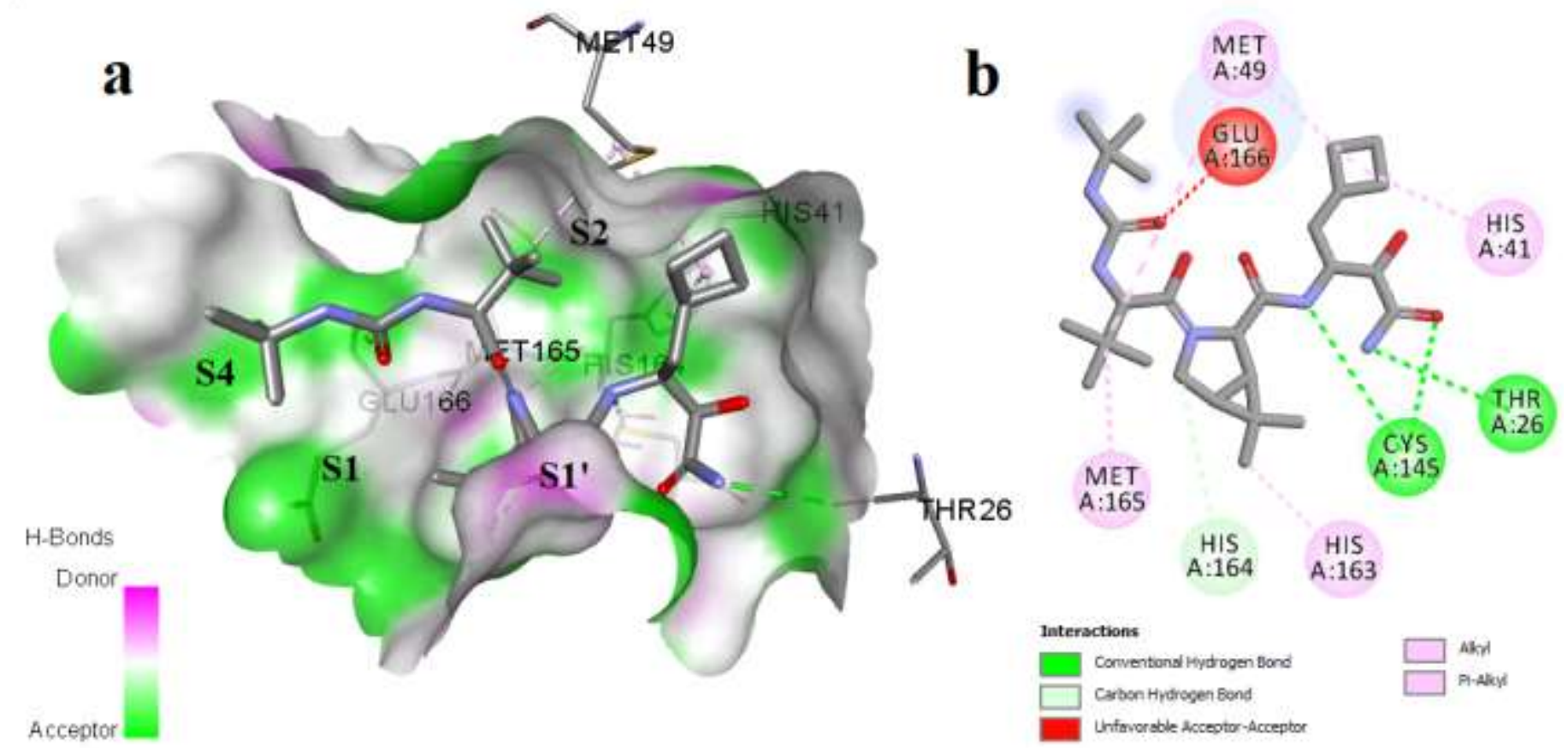

Figure 3. Bond interaction of boceprevir with mutant SARS-CoV-2 Mpro. a) Surface representation, b) Diagram representation 


\section{DISCUSSION}

$\mathrm{M}^{\text {pro }}$ cleaves polypeptide sequences after a glutamine residue, positioning the main protease as an ideal drug target because no human host-cell proteases are known with this substrate specificity [59]. The SARSCoV-2 Mpro consists of three basic domains. Domain I (residues 8-101) and domain II (residues 102-184) form a $\beta$-barrel structure. Domain III (residues 201-306) consists of $\alpha$-helices. Domains II and III are connected by a long loop (residues 185-200). Located in a cleft between domains I and II, the active site of $\mathrm{M}^{\text {pro }}$ contains a catalytic pair of conserved Cys 145 and His41 residues [22]. $\mathrm{M}^{\text {pro }}$ domains I and III are effective in functional movements and can undergo major structural changes.

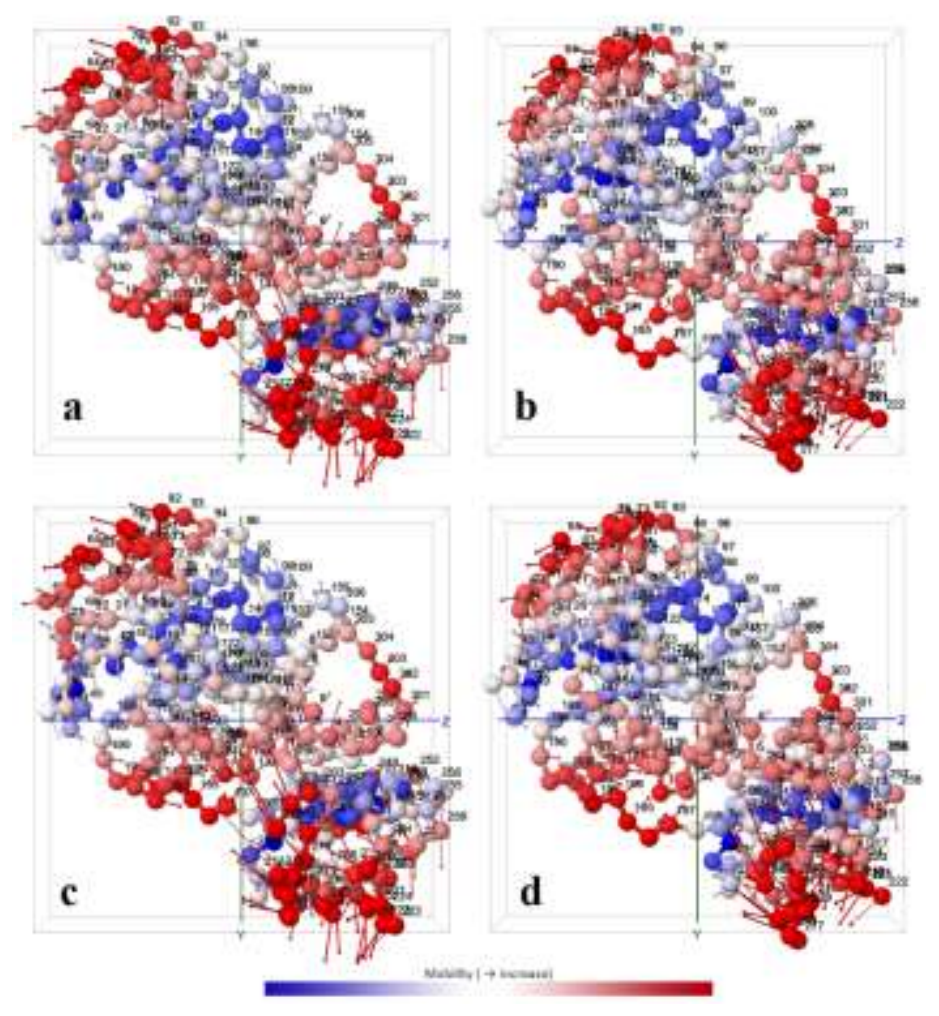

Figure 4. Slow mode collective motion of SARS-CoV-2 Mpro by Anisotropic network model. Vector ends indicate the direction of motion. Red represents mobile residues, white represents less mobile residues, white represents non-mobile

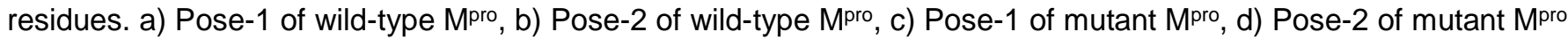

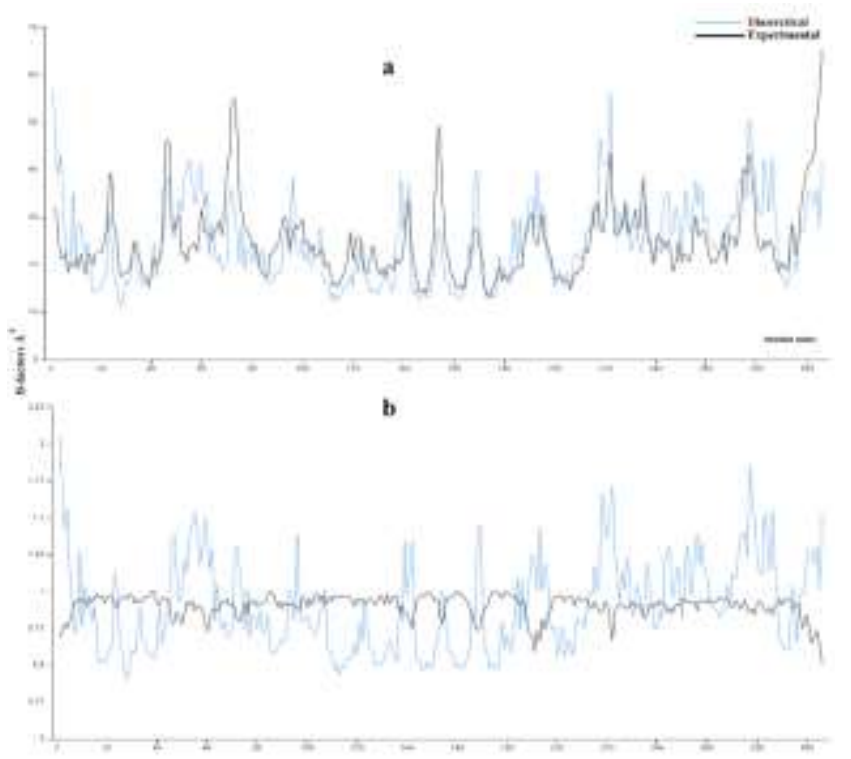


Figure 5. Root mean square fluctuations of residues by Gausian network model. a) Wild-type SARS-CoV-2 Mpro, b) Mutant SARS-CoV-2 Mpro

Boceprevir is an important protease inhibitor candidate for the SARS-CoV-2 $\mathrm{M}^{\text {pro }}$. In this study, we revealed that seven mutations detected in the $\mathrm{M}^{\text {pro }}$ of SARS-CoV-2 caused a decrease in the binding affinity of boceprevir. The mutations caused changes in the dynamic structure of $\mathrm{M}^{\text {pro }}$ and binding affinity of the inhibitor. The change in the bond number and formation with boceprevir in the mutant $\mathrm{M}^{\text {pro }}$ substrate binding pocket and catalytic region resulted in a decrease in affinity. It was observed that hydrogen bonding between boceprevir and the His41 residue in the catalytic region of the enzyme did not occur in the mutant $M^{\text {pro }}$ (Figures 2 and 3). The absence of Pro168-boceprevir hydrophobic alkyl interaction in the substrate binding pocket in the mutant protein affected the binding pattern. Khan et al. (2020) revealed that the Arg60Cys mutation detected in the $\mathrm{M}^{\text {pro }}$ in the Vietnam isolates of SARS-CoV-2 may cause a change in the dynamic structure of $\mathrm{M}^{\text {pro }}$ and a decrease in inhibitor affinity. Substitution of an amino acid with a charged side chain to an uncharged cysteine residue leads to loss of conserved ionic bond interaction, and the effect cascades to other conserved ionic interactions [10]. The interaction of boceprevir with the two catalytic residues (His41 and Cys145) of the enzyme is important for the inhibition of catalytic activity. Boceprevir interaction with the His163 and His164 residues in the substrate binding pocket, which plays an important role in catalytic activity, appears to exist in both wild-type and mutant $\mathrm{M}^{\text {pro }}$. The RMSF results showed the effect of mutations on dynamic fluctuations of residues. It was observed that fluctuation intervals were decreased in the mutant protein, while collective molecular fluctuation was preserved. Substrate recognition regions tend to exhibit structural flexibility to mediate substrate specificity [60]. It is thought that immobilization of key substrate binding site residues (His163, His164, Met165, and Glu166) and flexibility of residues (Ala191) near the binding site contribute to the realization of the bending (hinge) action for enzymatic activity $[22,24,61,62]$. Post-mutation reduction in flexibility at Pro108Ser (hinge region) and Ala191Val (near hinge, flexible region) residues may affect substrate binding and enzymatic activity (Table 2). The contribution of mutations in these two residues to the decreased ligand affinity is possible.

The Glu166 residue plays a role in dimerization, which is important for the catalytic activity of the enzyme.

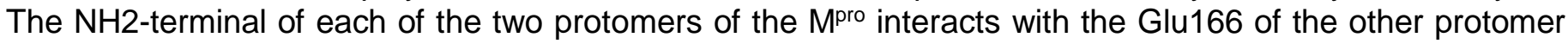
and helps shape the $S 1$ pocket of the substrate binding site and oxyanion hole [61,63-65]. In this study, it was observed that mutations transform the secondary structure between the 165 and 167 residues from coil to beta sheet. This change in the secondary structure may also affect dynamic fluctuation for Glu166 (Bfactor: $24.457-0.883 \AA^{2}$ ). It is thought that the changes caused by mutations may affect the enzyme's activeinactive conformational transformation and rearrangements in the substrate binding pocket.

The distance between His41:NE2 and Cys145:SG in the catalytic region in the mutant $\mathrm{M}^{\text {pro }}$ was positionally opened at $0.1 \AA$. Similarly, the distance between His41:CE1 and His 164:O opened at $0.2 \AA$. In the mutant $\mathrm{M}^{\text {pro }}$, the distance between His41:ND1 and His164:ND1 narrowed from 4.3 to $4.2 \AA$. The distance between His41:NE2 and Boc:O04 expanded from $2.7 \AA$ in wild-type $\mathrm{M}^{\text {pro }}$ to $3.6 \AA$ in mutant $\mathrm{M}^{\text {pro }}$ (Figure 6). After these positional changes, it was observed that the $\mathrm{H}$ bond interaction between His 41 and boceprevir disappeared in the mutant $\mathrm{M}^{\text {pro. }}$.

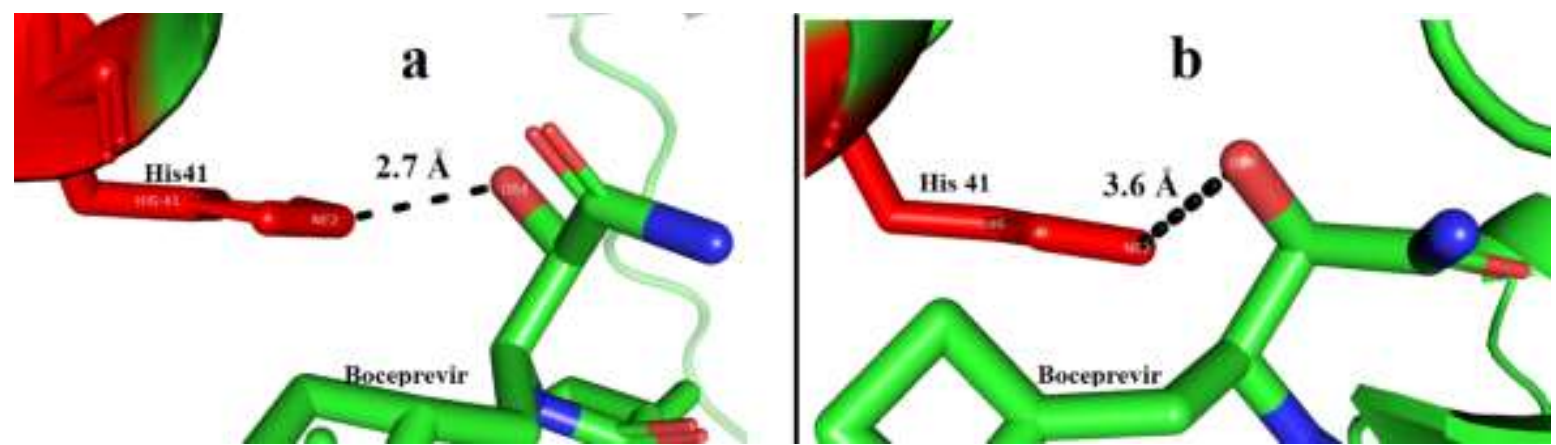

Figure 6. Hydrogen bond interaction of boceprevir with catalytic His41 residue of SARS-CoV-2 Mpro. a) Wild-type SARSCoV-2 Mpro, b) Mutant SARS-CoV-2 Mpro 
Cys 145 is known to play a role in the autocleavage of the $\mathrm{M}^{\text {pro }}$. The sulfur atom of the nucleophile Cys 145 , covalently bonded to the carbonyl carbon atom of the Gln306 residue in the C-terminal region, carries out autocleavage activity [65-68]. The preservation of the hydrogen bond between Cy145-Boc in both wild-type and mutant proteins despite the reduction in boceprevir affinity provides data that autocatalytic activity is inhibited and the conversion of $\mathrm{M}^{\text {pro }}$ to the dimer can be prevented.

Gly143 and His41 are the residues that are located in the $\mathrm{M}^{\text {pro }}$ binding pocket which is responsible for substrate stability by the hydrogen bonds they form [28,31]. While Gly143 hydrogen bond interaction is present in the wild protein, this interaction seems to disappear after mutations. It is seen that the hydrogen bond seen between His41 and boceprevir in the wild protein transforms into the pi-alkyl interaction, which is a weaker form of interaction. The change in the bond interaction in these 2 residues that provide proteaseboceprevir complex stabilization may be responsible for the decrease in boceprevir affinity.

The electrophilic a-ketoamide carbonyl appears to form a covalent bond with the sulfur of the catalytic Cys 145 and an S,O-acetale is formed. In Wild-type $\mathrm{M}^{\text {pro }}$, a-ketoamide oxygen forms hydrogen bonds with the main chain amides of Cys 145 and Gly143 that occupy the oxyanion hole [28,69]. Herein, it is His41 that controls all congruence. In the mutant protein, it is seen that the hydrogen bonding interaction of Gly 143 is eliminated and the formation is disrupted. The elimination of the Gly143 interaction occupying the oxyanion hole and the weakening of His41's interaction may affect the catalytic protonation of His 41 and Cys 145 residues. It is possible that the catalytic activity of $\mathrm{M}^{\text {pro }}$ in its natural form, which is blocked by boceprevir, may be restored in the mutant protein and show catalytic activity, albeit reduced.

\section{CONCLUSION}

An increasing number of cases due to transmission from person to person will cause new mutations to occur. The mutations in the SARS-CoV-2 $\mathrm{M}^{\text {pro }}$ can have negative consequences, such as a high increase in catalytic activity or an undesirable decrease in inhibitor affinity. Each new mutation carries risks that will invalidate therapies developed specifically for important viral biological structures. In this study, it was determined that SARS-CoV-2 Mpro mutations can cause a decrease in the affinity of boceprevir, one of the important therapeutic inhibitors for COVID-19 treatment. The results of this study will provide important data for drug design studies targeting the SARS-CoV-2 $\mathrm{M}^{\text {pro. }}$. However, further in vitro and in vivo analyses around this mentioned compound are required to ascertain these suggestions.

Funding: This research received no external funding.

Conflicts of Interest: The author declare no conflict of interest.

Acknowledgment: The genome and proteome sequence data of SARS CoV-2 were obtained from Virus database of National Center Biotechnology Information.

\section{REFERENCES}

1. Dai W, Zhang B, Jiang XM, Su H, Li J, Zhao Y, et al. Structure-based design of antiviral drug candidates targeting the SARS-CoV-2 main protease. Science. 2020;368(6497):1331-5.

2. Zhu N, Zhang D, Wang W, Li X, Yang B, Song J, et al. A novel coronavirus from patients with pneumonia in China, 2019. N Engl J Med. 2020;382(8):727-33.

3. Worldometer. Coronavirus Cases [Internet]. Worldometer. 2020 [cited 2020 May 11]. p. 1-22. Available from: https://www.worldometers.info/coronavirus/coronavirus-cases/\#daily-cases

4. Su S, Wong G, Shi W, Liu J, Lai ACK, Zhou J, et al. Epidemiology, genetic recombination, and pathogenesis of Coronaviruses. Trends Microbiol. 2016;24(6):490-502.

5. Cui J, Li F, Shi ZL. Origin and evolution of pathogenic coronaviruses. Nat Rev Microbiol. 2019;17(3):181-92.

6. Wong G, Liu W, Liu Y, Zhou B, Bi Y, Gao GF. MERS, SARS, and Ebola: the role of super-spreaders in infectious disease. Cell Host Microbe. 2015;18(4):398-401.

7. Zhang C, Huang S, Zheng F, Dai Y. Controversial treatments: an updated understanding of the Coronavirus Disease 2019. J Med Virol. 2020;92:1441-8.

8. Li G, De Clercq E. Therapeutic options for the 2019 novel coronavirus (2019-nCoV). Nat Rev Drug Discov. 2020;19(3):149-50.

9. Wu C, Liu Y, Yang Y, Zhang P, Zhong W, Wang Y, et al. Analysis of therapeutic targets for SARS-CoV-2 and discovery of potential drugs by computational methods. Acta Pharm Sin B. 2020;10(5):766-88. 
10. Khan MI, Khan ZA, Baig MH, Ahmad I, Farouk AEA, Song YG, et al. Comparative genome analysis of novel coronavirus (SARS-CoV-2) from different geographical locations and the effect of mutations on major target proteins: An in-silico insight. Plos One. 2020;15(9):1-18.

11. Callaway E. The coronavirus is mutating - does it matter? Nature. 2020;585(7824):174-7.

12. Chen J, Wang R, Wang M, Wei GW. Mutations strengthened SARS-CoV-2 infectivity. J Mol Biol. 2020;432(19):5212-26.

13. Akbulut E. SARS CoV-2 spike glycoprotein mutations and changes in protein structure. Trak Univ J Nat Sci. $2021 ; 22(1): 1-11$.

14. Wu F, Zhao S, Yu B, Chen YM, Wang W, Song ZG, et al. A new coronavirus associated with human respiratory disease in China. Nature. 2020;579(7798):265-9.

15. Baltimore D. Expression of animal virus genomes. Bacteriol Rev. 1971 Sep;35(3):235-41.

16. Ziebuhr J, Heusipp G, Siddell SG. Biosynthesis, purification, and characterization of the human coronavirus $229 \mathrm{E}$ 3C-like proteinase. J Virol. 1997;71(5):3992-7.

17. Ziebuhr J, Snijder EJ, Gorbalenya AE. Virus-encoded proteinases and proteolytic processing in the Nidovirales. J Gen Virol. 2000;81(4):853-79.

18. Chen Y, Liu Q, Guo D. Emerging coronaviruses: Genome structure, replication, and pathogenesis. J Med Virol. 2020;92(4):418-23.

19. Ullrich S, Nitsche C. The SARS-CoV-2 main protease as drug target. Bioorganic Med Chem Lett. 2020;30(17):1-8.

20. Poduri R, Joshi G, Jagadeesh G. Drugs targeting various stages of the SARS-CoV-2 life cycle: Exploring promising drugs for the treatment of Covid-19. Cell Signal. 2020;74(109721):1-20.

21. Jia Y, Shen G, Zhang Y, Huang K-S, Ho H-Y, Hor W-S, et al. Analysis of the mutation dynamics of SARS-CoV-2 reveals the spread history and emergence of RBD mutant with lower ACE2 binding affinity. bioRxiv [Internet]. 2020;2020.04.09.034942. Available from: https://www.biorxiv.org/content/10.1101/2020.04.09.034942v1

22. Tan J, Verschueren KHG, Anand K, Shen J, Yang M, Xu Y, et al. pH-dependent conformational flexibility of the SARS-CoV main proteinase (Mpro) dimer: Molecular dynamics simulations and multiple $\mathrm{X}$-ray structure analyses. $J$ Mol Biol. 2005;354(1):25-40.

23. Ho BL, Cheng SC, Shi L, Wang TY, Ho KI, Chou CY. Critical assessment of the important residues involved in the dimerization and catalysis of MERS Coronavirus Main Protease. PLoS One. 2015;10(12):1-18.

24. Zhang L, Lin D, Sun X, Curth U, Drosten C, Sauerhering L, et al. Crystal structure of SARS-CoV-2 main protease provides a basis for design of improved a-ketoamide inhibitors. Science (80- ). 2020;368(6489):409-12.

25. Fu L, Ye F, Feng Y, Yu F, Wang Q, Wu Y, et al. Both Boceprevir and GC376 efficaciously inhibit SARS-CoV-2 by targeting its main protease. Nat Commun. 2020;11(1):1-8.

26. Poordad F, McCone Jr J, Bacon BR, Bruno S, Manns MP, Sulkowski MS, et al. Boceprevir for untreated chronic HCV genotype 1 infection. N Engl J Med. 2011;364(13):1195-206.

27. Bacon BR, Gordon SC, Lawitz E, Marcellin P, Vierling JM, Zeuzem S, et al. Boceprevir for previously treated chronic HCV genotype 1 infection. N Engl J Med. 2011;364(13):1207-17.

28. Oerlemans R, Moreno AJR, Cong Y, Kumar ND, et al. Repurposing the HCV NS3-4A protease drug boceprevir as COVID-19 therapeutics. RSC Med. Chem. 2021;12(3):370-9.

29. Baker JD, Ulrich RL, Kraemer GC, Love JE, Kraemer BC. A drug repurposing screen identifies hepatitis $C$ antivirals as inhibitors of the SARS-CoV2 main protease. Plos One. 2021;16(2):1-13.

30. Mandour YM, Zlotos DP, Salem MA. A multi-stage virtual screening of FDA-approved drugs reveals potential inhibitors of SARS-CoV-2 main protease. J. Biomol. Struct. Dyn. 2020;23:1-12

31. Ma C, Sacco MD, Hurst B, Townsend JA, Hu Y, Szeto T, et al. Boceprevir, GC-376, and calpain inhibitors II, XII inhibit SARS-CoV-2 viral replication by targeting the viral main protease. Cell Res. 2020;30(8):678-92.

32. Sharma J, Bhardwaj VK, Singh R, Rajendran V, Purohit R, Kumar S. An in-silico evaluation of different bioactive molecules of tea for their inhibition potency against non-structural protein-15 of SARS-CoV-2. Food Chem. 2021;346(128933):1-9.

33. Bhardwaj VK, Singh R, Das $P$, Purohit R. Evaluation of acridinedione analogs as potential SARS-CoV-2 main protease inhibitors and their comparison with repurposed anti-viral drugs. Compu Biol Med. 2021;28(104117):1-13.

34. Bhardwaj VK, Singh R, Sharma J, Vidya R, Purohit R, Kumar S. Identification of bioactive molecules from tea plant as SARS-CoV-2 main protease inhibitors. J Biomol Struct Dyn. 2020;20:1-10.

35. Katoh K. MAFFT: a novel method for rapid multiple sequence alignment based on fast Fourier transform. Nucleic Acids Res. 2002;30(14):3059-66.

36. Carroll H, Beckstead W, O'Connor T, Ebbert M, Clement M, Snell Q, et al. DNA reference alignment benchmarks based on tertiary structure of encoded proteins. Bioinformatics. 2007;23(19):2648-9. 
37. Katoh K, Rozewicki J, Yamada KD. MAFFT online service: Multiple sequence alignment, interactive sequence choice and visualization. Brief Bioinform. 2018;20(4):1160-6.

38. Mount DW. Using BLOSUM in sequence alignments. Cold Spring Harb Protoc. 2008;3(6):pdb-top39.

39. Martin DP, Murrell B, Golden M, Khoosal A, Muhire B. RDP4: Detection and analysis of recombination patterns in virus genomes. Virus Evol. 2015;1(1).

40. Kumar S, Stecher G, Li M, Knyaz C, Tamura K. MEGA X: Molecular evolutionary genetics analysis across computing platforms. Mol Biol Evol. 2018;35(6):1547-9.

41. Waterhouse A, Bertoni M, Bienert S, Studer G, Tauriello G, Gumienny R, et al. SWISS-MODEL: Homology modelling of protein structures and complexes. Nucleic Acids Res. 2018;46(W1):296-303.

42. Guex N, Peitsch MC, Schwede T. Automated comparative protein structure modeling with SWISS-MODEL and Swiss-PdbViewer: A historical perspective. Electrophoresis. 2009;30(1):162-73.

43. Chen VB, Arendall WB, Headd JJ, Keedy DA, Immormino RM, Kapral GJ, et al. MolProbity: All-atom structure validation for macromolecular crystallography. Acta Crystallogr Sect D Biol Crystallogr. 2010;66(1):12-21.

44. Benkert $P$, Biasini M, Schwede T. Toward the estimation of the absolute quality of individual protein structure models. Bioinformatics. 2011;27(3):343-50.

45. Wiederstein M, Sippl MJ. ProSA-web: Interactive web service for the recognition of errors in three-dimensional structures of proteins. Nucleic Acids Res. 2007.

46. Buchan DWA, Minneci F, Nugent TCO, Bryson K, Jones DT. Scalable web services for the PSIPRED Protein Analysis Workbench. Nucleic Acids Res. 2013;41:349-57.

47. Pettersen EF, Goddard TD, Huang CC, Couch GS, Greenblatt DM, Meng EC, et al. UCSF Chimera - a visualization system for exploratory research and analysis. J Comput Chem. 2004;25(13):1605-12.

48. Zhang Y, Skolnick J. Scoring function for automated assessment of protein structure template quality. Proteins Struct Funct Genet. 2004;57(4):702-10.

49. Xu J, Zhang Y. How significant is a protein structure similarity with TM-score $=0.5$ ? Bioinformatics. 2010;26(7):88995.

50. Steffen C, Thomas K, Huniar U, Hellweg A, Rubner O, Schroer A. AutoDock4 and AutoDockTools4: Automated docking with selective receptor flexibility. J Comput Chem. 2010;31(16):2967-70.

51. Morris GM, Goodsell DS, Halliday RS, Huey R, Hart WE, Belew RK, et al. Automated docking using a Lamarckian genetic algorithm and an empirical binding free energy function. J Comput Chem. 1998;19(14):1639-62.

52. Li H, Chang YY, Lee JY, Bahar I, Yang LW. DynOmics: Dynamics of structural proteome and beyond. Nucleic Acids Res. 2017;45(W1):374-80.

53. Ming D, Wall ME. Allostery in a coarse-grained model of protein dynamics. Phys Rev Lett. 2005;95(198103):1-4.

54. Rodrigues CHM, Pires DEV, Ascher DB. DynaMut2: Assessing changes in stability and flexibility upon single and multiple point missense mutations. Protein Sci. 2021;30:60-69.

55. Pandurangan AP, Montano BO, Ascher DB, Blundell TL. SDM: a server for predicting effects of mutations on protein stability. Nucleic Acids Res. 2017;45:229-35.

56. Pires DEV, Ascher DB, Blundell TL. mCSM: predicting the effects of mutations in proteins using graph-based signatures. Str. Bioinformatics. 2014;30(3):335-42.

57. Pires DEV, Ascher DB, Blundell TL. DUET: a server for predicting effects of mutations on protein stability using an integrated computational approach. Nucleic Acids Res. 2014;42:314-9.

58. Pires DEV, Blundell TL, Ascher DB. mCSM-lig: quantifying the effects of mutations on protein-small molecule affinity in genetic disease and emergence of drug resistance. Sci Rep. 2016;6(29575):1-8.

59. Joshi RS, Jagdale SS, Bansode SB, Shankar SS, Tellis MB, Pandya VK, et al. Discovery of potential multi-targetdirected ligands by targeting host-specific SARS-CoV-2 structurally conserved main protease. J Biomol Struct Dyn. 2020;1-16.

60. Sheik Amamuddy O, Verkhivker G, Tastan Bishop O. Impact of emerging mutations on the dynamic properties the SARS-CoV-2 main protease: an in silico investigation. J Chem Inf Model. 2020;1-28.

61. Douangamath A, Fearon D, Gehrtz P, Krojer T, Lukacik P, Owen CD, et al. Crystallographic and electrophilic fragment screening of the SARS-CoV-2 main protease. Nat Commun. 2020;11(1):1-11.

62. Nguyen DD, Gao K, Chen J, Wang R, Wei GW. Unveiling the molecular mechanism of SARS-CoV-2 main protease inhibition from 137 crystal structures using algebraic topology and deep learning. Chem Sci. 2020;11(44):1203646.

63. Yang H, Yang M, Ding Y, Liu Y, Lou Z, Zhou Z, et al. The crystal structures of severe acute respiratory syndrome virus main protease and its complex with an inhibitor. Proc Natl Acad Sci U S A. 2003;100(23):13190-5.

64. Anand K, Palm GJ, Mesters JR, Siddell SG, Ziebuhr J, Hilgenfeld R. Structure of coronavirus main proteinase reveals combination of a chymotrypsin fold with an extra $\alpha$-helical domain. EMBO J. 2002;21(13):3213-24. 
65. Hilgenfeld R. From SARS to MERS: crystallographic studies on coronaviral proteases enable antiviral drug design. FEBS J. 2014;281(18):4085-96.

66. Lee J, Worrall LJ, Vuckovic M, Rosell FI, Gentile F, Ton AT, et al. Crystallographic structure of wild-type SARSCoV-2 main protease acyl-enzyme intermediate with physiological C-terminal autoprocessing site. Nat Commun. 2020;11(1):1-9.

67. Goyal B, Goyal D. Targeting the Dimerization of the Main Protease of Coronaviruses: A Potential Broad-Spectrum Therapeutic Strategy. ACS Comb Sci. 2020;22(6):297-305.

68. Kneller DW, Phillips G, O'Neill HM, Jedrzejczak R, Stols L, Langan P, et al. Structural plasticity of SARS-CoV-2 3CL Mpro active site cavity revealed by room temperature X-ray crystallography. Nat Commun. 2020;11(1):1-11.

69. Kumaran D, Andi B, Kreitler DF, Soares AS, Shi W, et al. Crystal structure of SARS-CoV-2 main protease (3CLpro/Mpro) in complex with covalent inhibitor boceprevir at $1.35 \mathrm{~A}$ resolution. PDB Entry 7K40. DOI: 10.2210/pdb7k40/pdb.

(C) (5) 52021 by the authors. Submitted for possible open access publication under the terms and BY NC (https://creativecommons.org/licenses/by-nc/4.0/). 